\title{
Diseño y construcción de un banco de ensayo para el estudio de pérdidas de carga por fricción y singularidad
}

\author{
Designed, built and evaluated hydraulic test bench \\ for studying head losses
}

David Ascencios T. ${ }^{1}$; Rafael Poma S. ${ }^{2}$

\begin{abstract}
Resumen
En el presente trabajo se diseña, construye y evalúa un banco de pruebas hidráulico para el estudio de pérdidas de carga en elementos de un sistema de riego, el cual permitirá que los estudiantes internalicen los conceptos hidráulicos, para la elaboración de diseño de sistemas de redes a presión. El banco de pruebas está compuesto por una estructura de soporte, dos electrobombas centrífugas de $0,85 \mathrm{Hp}$, cinco líneas de conducción de agua (cada línea compuesta de diferentes singularidades para su respectiva evaluación), una válvula volumétrica y un recipiente de almacenamiento de agua con una capacidad de 350 l. El equipo de bombeo consta de accesorios que hacen posible la configuración en serie o en paralelo de la bomba, que brindan al banco de pruebas mayor presión o caudal, según los elementos a evaluar. El caudal de agua se mide con un caudalímetro electrónico de 1". En la primera línea, se evalúa las pérdidas de carga por fricción en tuberías. En la segunda línea, se evalúa las pérdidas de carga en una válvula y las pérdidas de energía en una expansión y reducción con diferentes diámetros. En la tercera línea, se observa el comportamiento de válvulas hidráulicas con pilotos reductores y sostenedores de presión. En la cuarta línea, se evalúa la pérdida de carga en el Venturi y el filtro de anillos. En la quinta línea, se evalúa la presión en una red en paralelo y en serie, así como las pérdidas en singularidades como tees, codos y válvulas esféricas. En la evaluación de las tuberías, se calcula las pérdidas de carga teóricas mediante las fórmulas de Darcy-Weisbach, Hazen-Williams y Blasius y se compara con los resultados prácticos obtenidos con el banco de pruebas, de estas tres se elige la fórmula más adecuada utilizando la de equilibrio de NASH. En la evaluación de las singularidades, se determina la curva caudal vs pérdida de carga. El costo del banco de prueba es de S/. 5.500,00.
\end{abstract}

Palabras clave: banco de pruebas; pérdidas por fricción; hidráulica; válvulas.

\begin{abstract}
In the present work we designed, built and evaluated a hydraulic test bench for studying head losses in elements of an irrigation system, which will allow students to internalize hydraulic concepts for the development of networks systems designs of pressurized irrigation systems. The test bench consists of a supporting structure, two centrifugal pumps of $0,85 \mathrm{Hp}, 5$ water transmission lines (each line composed of different singularities for its evaluation), a volumetric valve and a water storage container with a capacity of 3501 . The pumping equipment consists of accessories which permit configuration in series or parallel, which provide the test bench greater pressure or flow, according to the elements to evaluate. The water flow was measured with an electronic flow meter of 1". In the first line it's evaluated the friction head losses in pipes. On the second line it's evaluated the head losses in a valve and energy losses in expansion and reduction with different diameters. The third line shows the behavior of hydraulic valves with pilot pressure reducers and holders of pressure. On the fourth line it's evaluated the head losses in the Venturi and the filter of rings. In the fifth line pressure it's evaluated the pressure on a network pipe in parallel and in series, also head losses in singularities as "tees", elbows, and ball valves. In the evaluation of pipes, theoretical head losses are calculated by Darcy-Weisbach, Hazen-Williams and Blasius formulas and is compared with practical results obtained with the test bench. The most appropriate formula is chosen by using NASH equilibrium formula. In the evaluation of singularities the curve of flow vs head losses of head was determined. The cost of the test bench is S/. 5.500,00.
\end{abstract}

Keywords: test bench; head losses; hydraulic; valves.

1. Universidad Nacional Agraria La Molina, Lima (Perú). Email: dascencios@lamolina.edu.pe

2. Irrigaciones SAC, Universidad Nacional Agraria La Molina, Lima (Perú). Email: rapso_piscis@hotmail.com 


\section{Introducciòn}

En el análisis y diseño de las instalaciones hidráulicas, es necesario conocer los conceptos que relacionan los cambios de energía hidráulica en el fluido al atravesar un elemento, con un caudal dado. Existen diversos sistemas de transporte de fluidos compuestos por tuberías y singularidades de diferentes materiales y diámetros que tienen una extensa aplicación en plantas de procesos, proyectos de riego presurizado y sistemas de suministro de agua para las ciudades. En el diseño de redes de tuberías, el análisis debe realizarse en base a cálculos de las pérdidas de energía entre dos puntos del sistema de flujo, producidos por la fricción que se genera cuando un líquido fluye a través de tuberías, válvulas, uniones, codos y otros elementos.

En las prácticas de hidráulica de tuberías, los estudiantes captan con dificultad los conceptos relacionados a los cambios de energía hidráulica debido a la "pérdida de carga", porque no se observa físicamente el fenómeno, debido a la falta de bancos de prueba experimentales y la falta de equipos en los laboratorios, por lo que es necesario contar con un banco de pruebas que permita visualizar, manipular y experimentar los cambios que sufren los fluidos al modificar las variables que están directamente relacionadas con su comportamiento, como lo son el diámetro, el caudal, el material, cantidad y calidad. En el presente trabajo se diseña, construye y evalúa un banco de pruebas hidráulico para el estudio de pérdidas de carga en ciertos elementos de diferentes materiales ( $\mathrm{PVC}, \mathrm{PE}, \mathrm{F}^{\circ} \mathrm{G}^{\circ}$ ) dentro de los rangos de trabajo del banco (caudales de $0,1-1,11 / \mathrm{s}$, presiones de 5 a 60 metros), para demostrar a los estudiantes los fenómenos que ocurren en las redes de tuberías y darles la oportunidad de interactuar con ellos modificando las variables involucradas.

\section{Materiales y métodos}

Los materiales y equipos para la construcción del banco de pruebas son principalmente, las bombas centrifugas, válvulas manuales hidráulicas con pilotos de control, filtros, tubo venturi, tuberías de PVC y PE, planchas y tubos de fierro, accesorios como codos, tees, etc. A continuación, consignamos las actividades desarrolladas para el diseño y evaluación del banco.

\section{Fase de laboratorio}

\section{Diseño del banco de pruebas}

El banco de pruebas está conformado por tuberías lisas y rugosas de $\mathrm{PVC}, \mathrm{PE} \mathrm{y} \mathrm{F}^{\circ} \mathrm{G}^{\circ}$, válvulas hidráulicas con piloto reductor y sostenedor de presión, válvulas plasson, válvulas esféricas, tubo de venturi, filtro de anillos, singularidades y puntos de prueba de presión. En la parte inferior tiene una bandeja de metal sobre la cual se recolecta el agua que sale del banco y que es llevada al depósito de agua para su recirculación (Fig. 1).

\section{Construcción del banco de pruebas}

Una vez realizado el diseño, se procedió con la construcción de la estructura de soporte del banco y la bandeja; posteriormente, se procedió con el armado y colocado de tuberías, válvulas y demás accesorios.

\section{Evaluación del banco de pruebas}

Se realizaron las pruebas en los siguientes elementos: Tuberías PVC, válvula de asiento inclinado, expansión y reducción con transición en tuberías lisas, válvula hidráulica con piloto sostenedor de presión, válvula hidráulica con piloto reductor de presión, tubo venturi, filtro de anillos, redes en paralelo y en serie y singularidades.

\section{Fase de Gabinete}

En esta fase, se procesa la información obtenida en el laboratorio, se utiliza los datos y mediciones tomadas en el banco de pruebas. Estos datos fueron principalmente de presión y caudal. Para el procesamiento automático de datos, se trabajó en una hoja de cálculo de excel, la cual nos permitió realizar las evaluaciones de diversas expresiones y fórmulas empleadas. Los resultados de las evaluaciones de las pérdidas de carga en las tuberías y accesorios son los coeficientes de fricción y las curvas de

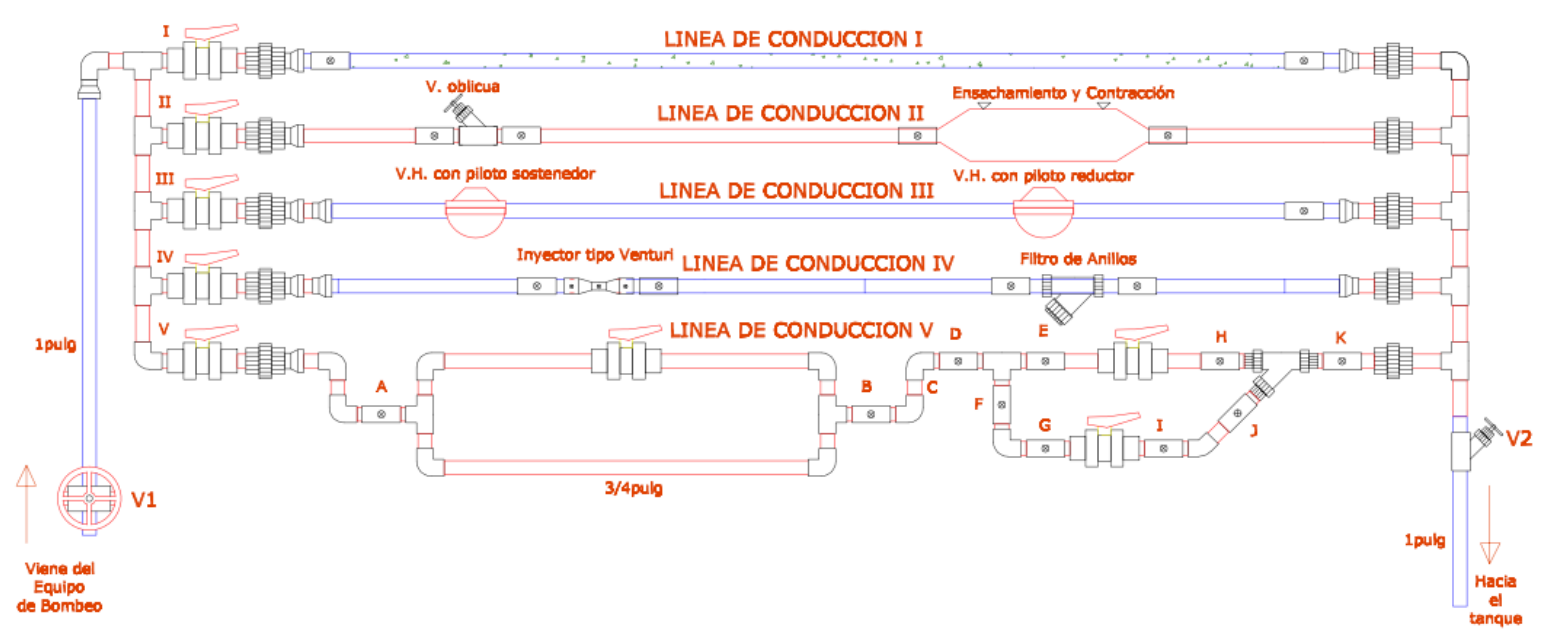

Figura 1. Esquema de banco de prueba. 
pérdida de carga vs caudal, respectivamente. Los valores de pérdidas de cargas obtenidas experimentalmente son comparadas con las calculadas matemáticamente a partir de fórmulas empíricas como son: Darcy-Weisbach, Hazen-Williams y Blasius.

\section{Resultados y discusión}

\section{Descripción del banco de pruebas}

\section{Equipo de bombeo}

Se utilizó el equipo existente en el Laboratorio de Riego y Drenaje del Departamento de Recursos Hídricos, conformado por dos electrobombas de las siguientes características: Marca PEDROLLO, modelo CPm 610x, tipo centrífuga, con una velocidad de $3450 \mathrm{rpm}$, potencia 0,85 HP, presión 30-13 metros, caudal 10-70 L/min, amperaje de $4 \mathrm{Amp}$, voltaje de $220 \mathrm{~V}$. Las conexiones entre las bombas permiten hacerlas trabajar simultáneamente en serie o en paralelo para lograr presiones o caudales altos, respectivamente. Las tuberías usadas para estas conexiones son de PVC C-10 de 1" de diámetro. Para medir el caudal se utilizó un caudalímetro, instalado a la salida del equipo de bombeo, tomando como unidad de medida litros/minuto.

\section{Depósito de agua}

Está conformado por un depósito de metal de $60 \mathrm{~cm} \mathrm{x}$ $100 \mathrm{~cm}$ de base con una altura de $65 \mathrm{~cm}$ para una capacidad aproximada de 3501 .

\section{Estructura de soporte del banco de pruebas}

Se construyó una estructura móvil para soportar el banco de pruebas y las vibraciones generadas al ponerlo en funcionamiento. Para ello, se utilizaron tubos de fierro y planchas de metal que permiten captar el agua que sale del banco y devolverlo al depósito de agua. La dimensión de esta estructura es de $2,50 \mathrm{~m} \times 1,30 \mathrm{~m} \times 1,20 \mathrm{~m}$ de alto.

\section{Elementos del banco de pruebas}

\section{Conducción y distribución del agua}

La unidad de conducción de agua consta de dos partes: la de entrada, que consiste en una tubería de PVC de 1" de diámetro en cuya parte inicial lleva una unión universal para el fácil empalme a la unidad de bombeo, y la de la salida con tuberías y accesorios de $3 / 4$ " de diámetro y una válvula de asiento inclinado de $3 / 4$ " de diámetro para la regulación de la presión en el banco. Además, se colocó una manguera de polietileno de $25 \mathrm{~mm}$, con una acople $3 / 4$ " a $25 \mathrm{~mm}$ para el desfogue de agua al depósito y ser después nuevamente bombeada al sistema.

Para la distribución del agua, se utilizaron cinco válvulas esféricas de PVC de 3/4" diámetro, que independizan el flujo en cada línea evaluada. Se usó, además, tuberías de $3 / 4$ ", 4 codos de $3 / 4$ ", 8 tees de $3 / 4$ ", un codo de 1 "y una reducción de 1 " a $3 / 4$ " de diámetro para las conexiones entre las válvulas y toda la unidad de conducción (Figs. 1 y 2 ).

\section{Puntos de prueba de presión}

Los puntos de prueba de presión se colocaron al inicio y al final de cada tubería y a la entrada y salida de cada accesorio o elemento singular. Los puntos de prueba se conformaron por collarines de PE de $32 \mathrm{~mm} \mathrm{x} \mathrm{1/2"} \mathrm{y} 25 \mathrm{~mm}$ $\mathrm{x} 1 / 2$ " (según el diámetro de la tubería), bushing de $1 / 2$ "- 4" y tomas manométricas tipo aguja de 4 " y de 8 ".

\section{Líneas de evaluación de elementos \\ Línea de evaluación I}

Se armaron tres líneas de conducción portátiles, con uniones universales de $3 / 4$ " de diámetro al inicio y al final de cada línea para el fácil ensamblaje o desensamblaje. Las líneas se conformaron con tuberías de PVC C-10 de 1 ", $3 / 4 ", 1 / 2 "$ de diámetro, con interiores lisos, con una longitud de $1,80 \mathrm{~m}$ cada una; se usaron además dos reducciones de 1 "- 3/4" y dos reducciones de 3/4" a $1 / 2$ " de

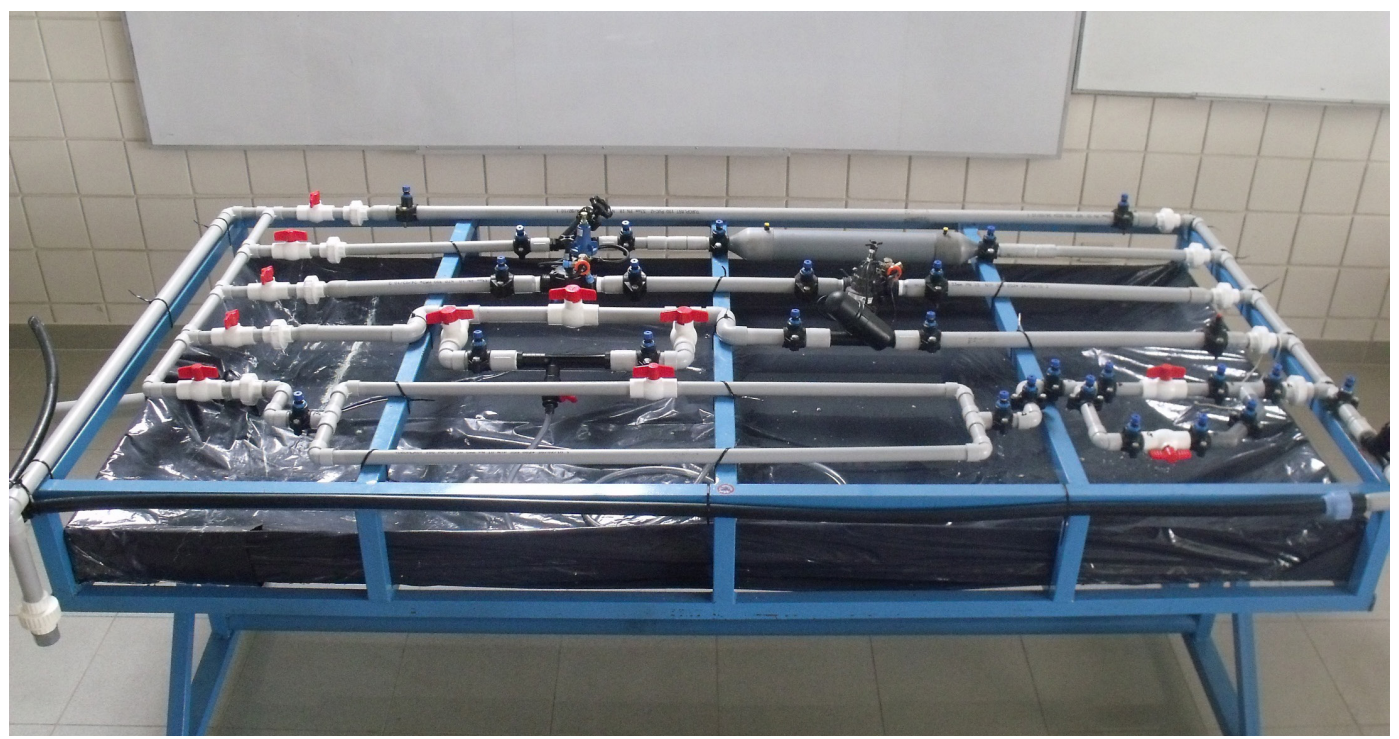

Figura 2. Conducción y distribución del agua. 
diámetro. Se instalaron dos puntos de prueba de presión por cada tubería, separados por 1,80m entre sí.

\section{Línea de evaluación II}

Se armó una línea de conducción portátil con uniones universales de $3 / 4$ " de diámetro al inicio y al final de cada línea para el fácil ensamblaje. La línea se conformó con una tubería de PVC C-10 de $3 / 4$ " de diámetro, una válvula de asiento inclinado de $3 / 4$ ", dos reducciones de $2 \frac{1}{2}$ " a $3 / 4$ ", un niple de $2 \frac{1}{2}$ " de diámetro, dos uniones mixtas de $3 / 4$ " y dos uniones de simple presión de $3 / 4$ ". Se colocaron cuatro puntos de prueba de presión de $1 / 4$ ", antes y después de la válvula y de las reducciones $21 / 2$ " a $3 / 4$ ", que unen el niple de $21 / 2$ "y dos puntos de prueba de $1 / 8$ " sobre el niple de $2 \frac{1}{2}$ " a inicio y al final de este.

\section{Línea de Evaluación III}

Se armó una línea de conducción portátil con uniones universales de $3 / 4$ " de diámetro al inicio y al final de cada línea para el fácil ensamblaje. La línea se conformó con una tubería de PVC C-10 de 1 " de diámetro con reducciones de 1 " a $3 / 4$ " al inicio y al final de este tramo, dos válvulas hidráulicas de 1", un piloto sostenedor de presión y un piloto reductor de presión, estas últimas instaladas en sus respectivas válvulas hidráulicas.

\section{Línea de Evaluación IV}

Se armó una línea de conducción portátil con uniones universales de $3 / 4$ " de diámetro al inicio y al final de cada línea para el fácil ensamble. La línea se conformó con una tubería de PVC C-10 de 1 " de diámetro con reducciones de 1 " a $3 / 4$ " al inicio y al final de este tramo, un tubo de venturi de 1" de diámetro con manguera de succión y canastilla, tres válvulas esféricas de PVC de
1" de diámetro para la regulación del paso de agua por el venturi (puente), un filtro de anillos de 1"y de 120 mesh, cuatro uniones mixtas de 1", dos tees de 1"y seis codos de 1". Se colocaron cuatro puntos de prueba de presión, antes y después del tubo de venturi y el filtro de anillos.

\section{Línea de Evaluación $V$}

Se armó una línea de conducción portátil con uniones universales de $3 / 4$ " de diámetro al inicio y al final de cada línea para el fácil ensamblaje. La línea se conformó por una tubería de PVC C-10 de 3/4" de diámetro, tres válvulas esféricas de PVC de $3 / 4$ ", nueve codos de $3 / 4$ ", cuatro tees de $3 / 4$ " y dos codos de $3 / 4$ " x $45^{\circ}$ que conforman dos redes paralelas de tuberías y accesorios de conexión. Se colocaron 11 puntos de prueba de presión antes y después de cada red, así como de cada singularidad.

\section{Mandos hidráulicos para la apertura y cierre de válvulas hidráulicas}

Los mandos hidráulicos se ubicaron a la entrada del banco, la presión en este punto es mayor que en cualquier otro punto del banco de pruebas, necesario para el funcionamiento de los mandos. Se armó una línea de conducción con accesorios de fierro galvanizado de 1/4", a la que llamamos "Quena", y en donde se ubican las válvulas de tres vías. Desde estas válvulas en la entrada del banco se tendieron mangueras de PE de $8 \mathrm{~mm}$ hacia las válvulas de tres vías colocadas sobre las válvulas hidráulicas reductora y sostenedora de presión.

\section{Evaluación hidráulica}

Línea de evaluación I, tubería lisa de pvc de 3/4"

Se evaluó la pérdida de carga por fricción en un tramo de 1,80m de tubería lisa de PVC variando los caudales

Tabla 1. Pérdidas de carga en tuberías lisas de PVC de 3/4" x 1,80m

\begin{tabular}{|c|c|c|c|c|c|c|c|}
\hline \multirow[b]{2}{*}{ No } & \multirow{2}{*}{$\begin{array}{l}\text { CAUDAL } \\
(1 / \mathrm{min})\end{array}$} & \multicolumn{3}{|c|}{ PRESIÒN } & \multicolumn{3}{|c|}{ PÈRDIDAS DE CARGA TEORICAS (m) } \\
\hline & & $\begin{array}{c}\text { ENTRADA } \\
\text { (m) }\end{array}$ & $\begin{array}{l}\text { SALIDA } \\
(\mathrm{m})\end{array}$ & $\begin{array}{l}\Delta \mathbf{H} \\
(\mathbf{m})\end{array}$ & $\begin{array}{c}\text { DARCY - } \\
\text { WEISBACH }\end{array}$ & $\begin{array}{c}\text { HAZEN - } \\
\text { WILLIAMS }\end{array}$ & BLASIUS \\
\hline 1 & 10,6 & 4,30 & 4,30 & 0,00 & 0,01 & 0,02 & 0,02 \\
\hline 2 & 15,0 & 2,80 & 2,75 & 0,05 & 0,03 & 0,04 & 0,04 \\
\hline 3 & 20,1 & 2,80 & 2,75 & 0,05 & 0,05 & 0,06 & 0,07 \\
\hline 4 & 24,7 & 4,25 & 4,20 & 0,05 & 0,08 & 0,09 & 0,10 \\
\hline 5 & 29,6 & 4,90 & 4,80 & 0,10 & 0,11 & 0,13 & 0,14 \\
\hline 6 & 36,0 & 7,30 & 7,20 & 0,10 & 0,17 & 0,19 & 0,20 \\
\hline 7 & 40,5 & 5,80 & 5,60 & 0,20 & 0,22 & 0,23 & 0,25 \\
\hline 8 & 43,9 & 6,00 & 5,80 & 0,20 & 0,25 & 0,27 & 0,29 \\
\hline 9 & 45,8 & 3,00 & 2,70 & 0,30 & 0,28 & 0,29 & 0,31 \\
\hline 10 & 50,1 & 4,80 & 4,50 & 0,30 & 0,33 & 0,35 & 0,36 \\
\hline 11 & 54,6 & 5,20 & 4,80 & 0,40 & 0,39 & 0,41 & 0,42 \\
\hline 12 & 58,7 & 5,20 & 4,80 & 0,40 & 0,45 & 0,47 & 0,48 \\
\hline 13 & 59,8 & 6,80 & 6,40 & 0,40 & 0,47 & 0,48 & 0,49 \\
\hline 14 & 65,3 & 7,10 & 6,60 & 0,50 & 0,56 & 0,57 & 0,57 \\
\hline 15 & 71,4 & 7,60 & 7,00 & 0,60 & 0,67 & 0,67 & 0,67 \\
\hline
\end{tabular}


desde 10,6 1/min hasta 71,4 1/min. El diámetro externo e interno de dicha tubería fue de $26,5 \mathrm{~mm}$ y $22,9 \mathrm{~mm}$, respectivamente. La Tabla 1 muestra la variación de caudales con los que se trabajó en la prueba así como la presión a la entrada y salida del conducto y la pérdida de carga $(\Delta \mathrm{H})$ correspondiente para cada caudal. Se puede notar que las pérdidas de carga se hacen perceptibles a partir de los 15.0 1/min, y a medida que se van aumentando los caudales aumenta también la pérdida de carga debido al incremento de la fricción entre el fluido y las paredes del conducto. La máxima pérdida de carga $67 \mathrm{~cm}$ con un caudal de $71,41 / \mathrm{min}$ y una velocidad de flujo de $2,89 \mathrm{~m} / \mathrm{s}$. Por otra parte se aprecia también las pérdidas de carga teóricas obtenidas del cálculo con fórmulas empíricas: Darcy-Weisbach, Hazen-Williams y Blasius.

Para conocer la fórmula que presenta mejor aproximación a los valores de pérdidas de carga obtenidas experimentalmente, usamos la del equilibrio de NASH:

$$
\begin{aligned}
& \mathrm{NASH}=1-\quad \sum(\mathrm{Ob}-\mathrm{Ot})^{2} . \\
& \sum\left(\overline{\mathrm{ob}-0 \text { oprom })^{2}}\right.
\end{aligned}
$$

Donde:

$\mathrm{Ob} \quad$ : valores observados $(\Delta \mathrm{H}$ obtenido experimentalmente).

Ot: valores teóricos (pérdidas de carga teóricas).

Oprom : promedio aritmético de valores observados.

Valores de $\Delta \mathrm{H}$ medidos en el banco de prueba, con relación a los obtenidos de la fórmula de Darcy-Weisbach, $\mathrm{NASH}=0,945$

Valores de $\Delta H$ medidos en el banco de prueba, en relación a los obtenidos de la fórmula de Hazen-Williams, NASH $=0,915$

Valores de $\Delta \mathrm{H}$ medidos en el banco de prueba, en relación a los obtenidos de la fórmula de Blasius, NASH $=0,887$

De los datos obtenidos, se seleccionó el de mayor valor de NASH $=0,945$ que corresponde al de los valores obtenidos de la fórmula de Darcy-Weisbach. En la Fig. 3 se presenta lo afirmado en el párrafo anterior, al observar que los puntos de las pérdidas de carga obtenidos experimentalmente se acercan más a la curva de pérdida de carga calculada mediante la fórmula de Darcy-Weisbach.

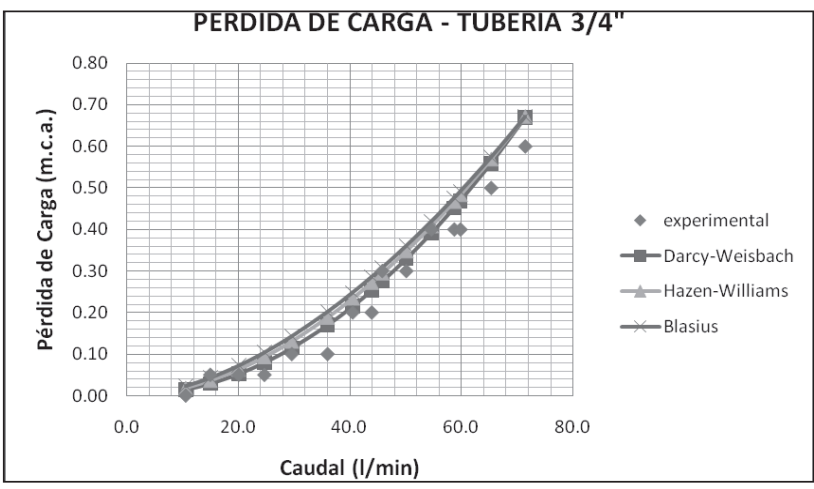

Figura 3. Curvas de pérdida de carga en tuberías de PVC 3/4" - teóricas y experimentales.

\section{Línea de evaluación II, válvula de asiento inclinado de $3 / 4 "$}

Se evaluó la pérdida de carga entre la entrada y salida de la válvula de polietileno completamente abierta, variando los caudales desde los 10,3 1/min hasta los 64,3 1/min.

En la Tabla 2 se muestra la variación de caudales con los que se trabajó en la prueba, así como la presión a la entrada y salida de la válvula y la pérdida de carga $(\Delta \mathrm{H})$ correspondiente para cada caudal, con la válvula completamente abierta. Se observa que las pérdidas de carga se hacen perceptibles a partir de los 10,9 1/min y a medida que van aumentando los caudales, aumentan también las pérdidas de carga debido a singularidad de la válvula.

Tabla 2. Pérdidas de carga en válvula de asiento inclinado de $3 / 4 "$ - abierta

\begin{tabular}{|c|c|c|c|c|}
\hline \multirow{2}{*}{ No } & Caudal & \multicolumn{3}{|c|}{ Presión } \\
\cline { 2 - 5 } & $\mathbf{( 1 / m i n )}$ & $\begin{array}{c}\text { Entrada } \\
(\mathbf{m})\end{array}$ & $\begin{array}{c}\text { Salida } \\
(\mathbf{m})\end{array}$ & $\begin{array}{c}\Delta \mathbf{H} \\
\mathbf{( m )}\end{array}$ \\
\hline 1 & 10,90 & 4,20 & 4,15 & 0,05 \\
\hline 2 & 19,80 & 6,60 & 6,50 & 0,10 \\
\hline 3 & 31,00 & 5,80 & 5,40 & 0,40 \\
\hline 4 & 40,00 & 6,60 & 6,00 & 0,60 \\
\hline 5 & 49,70 & 8,80 & 8,00 & 0,80 \\
\hline 6 & 59,60 & 7,90 & 6,80 & 1,10 \\
\hline 7 & 64,30 & 9,00 & 7,80 & 1,20 \\
\hline
\end{tabular}

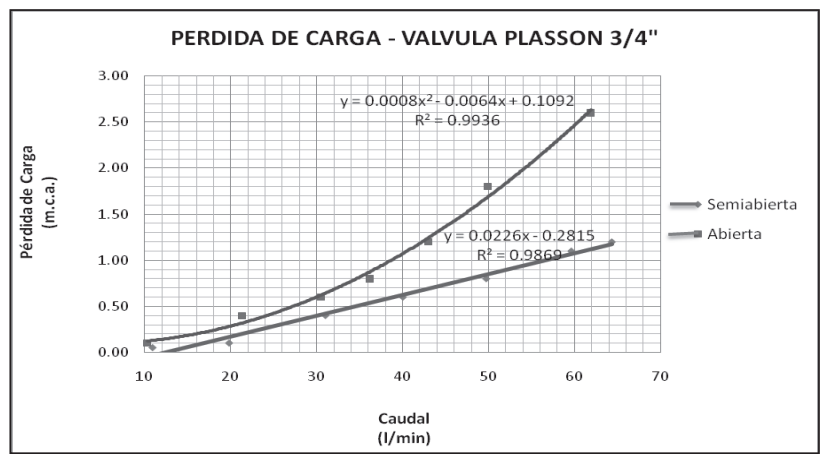

Figura 4. Curvas de pérdida de carga en válvula de asiento inclinado de $3 / 4$ " abierta.

La Fig. 4 muestra las curvas de variación de pérdidas de carga para cierto caudal en válvulas de asiento inclinado completamente abiertas y semiabiertas. La válvula semiabierta va a generar mayores pérdidas de carga que la válvula abierta, al hacer pasar la misma cantidad de agua. La curva generada por la válvula completamente abierta tiene una tendencia lineal con valor de $\mathrm{R}^{2}=0,9869$, regida por la ecuación: $\Delta \mathrm{H}=0,0226 . \mathrm{Q}-0,2816$. La curva generada por la válvula semiabierta presenta una tendencia polinomial de segundo grado con valor $\mathrm{R}^{2}=0,9936$, regida por la ecuación: $\Delta \mathrm{H}=0,0008 . \mathrm{Q}^{2}-0,0064 . \mathrm{Q}+0,1092$. 


\section{Tubería con expansión y reducción de 3/4" a 2 1/2"}

Se evaluó la pérdida de carga entre cuatro puntos de un tramo de tubería de PVC, con reducciones de $3 / 4$ " a $2 \frac{1}{2}$ " (diámetro externo) que representan un ensanchamiento y una contracción. El diámetro interno en los puntos (1) y (4) es de $16,75 \mathrm{~mm}$, mientras que en los puntos (2) y (3) es de $63,75 \mathrm{~mm}$.

La Tabla 3 presenta la variación de los caudales con los que se trabajó en la prueba, así como la presión en cada uno de los puntos del conducto y la diferencia de presión $(\Delta \mathrm{H})$ entre dos puntos. Se puede notar que la presión aumentó del punto (1) al punto (2) y disminuyó entre los puntos (3) y (4), (1) y (4), siendo la diferencia de esta última la pérdida de carga. Para los dos primeros casos hay que sumar a las lecturas las cargas de velocidad, que son diferentes entre cada punto debido a la diferencia de diámetros.

\section{Línea de evaluación III, válvula hidráulica 1"}

Se evaluó la pérdida de carga entre la entrada y salida de la válvula de polietileno, variando los caudales desde los 4,40 1/min hasta los 67,5 1/min.

En La Tabla 4 se muestra la variación de caudales, presiones de entrada y salida de la válvula y la pérdida de carga $(\Delta \mathrm{H})$ correspondiente para cada caudal. Se aprecia que al aumentar los caudales aumenta también la pérdida de carga debido a la válvula.

En la Fig. 5 se presenta la curva de variación de pérdidas de carga para ciertos caudales en válvulas hidráulicas de

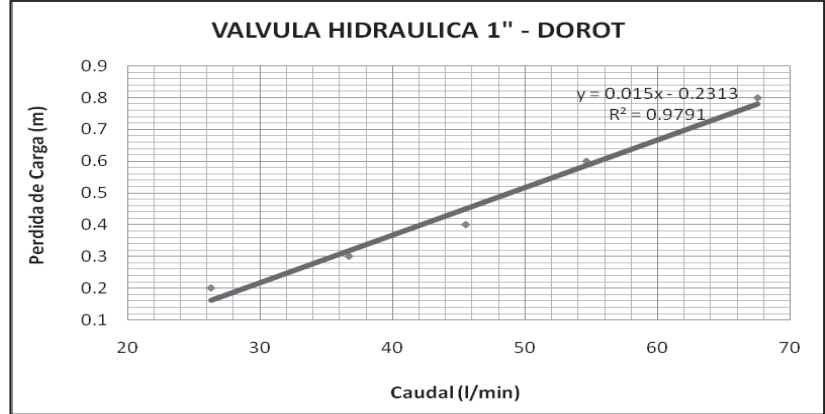

Figura 5. Curva de pérdidas de carga en válvula hidráulica de 1".

1". Las curva generada por la válvula completamente abierta tiene una tendencia lineal con valor de $\mathrm{R}^{2}=0,9791$, regida por la ecuación: $\Delta \mathrm{H}=0,015$.Q-0,2313.

Línea de evaluación IV, tubo de venturi de 1"

Se evaluó el caudal de succión del tubo de venturi a medida que se modifica la pérdida de carga entre la entrada y la salida del conducto.

En La Tabla 5 se presenta la variación de las presiones con las que se trabajó en la prueba, así como el caudal succionado por el venturi correspondiente para cada pérdida de carga. El tubo de venturi empieza a trabajar con una pérdida de carga de 7,00 m.c.a, succionando un caudal de 10,65 1/hr. A medida que las pérdidas de carga son mayores, aumenta el caudal de succión llegando a alcanzar su máximo caudal.

Tabla 3. Pérdidas de carga en conductos de PVC con expansión y reducción

\begin{tabular}{|c|c|c|c|c|c|c|c|c|}
\hline \multirow{2}{*}{ No } & \multirow{2}{*}{$\begin{array}{l}\text { CAUDAL } \\
\text { (1/min) }\end{array}$} & \multicolumn{4}{|c|}{ PRESIÓN } & \multicolumn{3}{|c|}{$\begin{array}{c}\text { DIFERENCIA DE PRESIÓN } \\
(\mathrm{m})\end{array}$} \\
\hline & & $\begin{array}{l}\text { PUNTO } 1 \\
\quad(\mathrm{~m})\end{array}$ & $\begin{array}{l}\text { PUNTO } 2 \\
\text { (m) }\end{array}$ & $\begin{array}{l}\text { PUNTO } 3 \\
\text { (m) }\end{array}$ & $\begin{array}{l}\text { PUNTO } 4 \\
\text { (m) }\end{array}$ & $\begin{array}{l}\Delta H \\
\text { (1) } y(2)\end{array}$ & $\begin{array}{l}\Delta H \\
\text { (3) } y(4)\end{array}$ & $\begin{array}{c}\Delta H \\
\text { (1) y (4) }\end{array}$ \\
\hline 2 & 18,40 & 4,00 & 4,00 & 4,00 & 3,90 & 000 & 0,10 & 0,10 \\
\hline 3 & 31,70 & 4,70 & 4,80 & 4,80 & 4,50 & $-0,10$ & 0,30 & 0,20 \\
\hline 4 & 40,60 & 3,70 & 3,80 & 3,80 & 3,30 & $-0,10$ & 0,50 & 0,40 \\
\hline 5 & 51,50 & 2,40 & 2,60 & 2,60 & 1,80 & $-0,20$ & 0,80 & 0,60 \\
\hline 6 & 60,30 & 3,50 & 3,90 & 3,90 & 2,80 & $-0,40$ & 1,10 & 0,70 \\
\hline 7 & 65,00 & 4,20 & 4,60 & 4,60 & 3,30 & $-0,40$ & 1,40 & 0,90 \\
\hline
\end{tabular}

Tabla 4. Pérdidas de carga válvula hidráulica plástica de 1"

\begin{tabular}{|c|c|c|c|c|}
\hline \multirow{2}{*}{ No } & \multicolumn{2}{|c|}{ CAUDAL } & \multicolumn{3}{|c|}{ PRESIÓN } \\
\cline { 2 - 5 } & $\mathbf{( 1 / m i n )}$ & $\begin{array}{c}\text { ENTRADA } \\
(\mathbf{m})\end{array}$ & $\begin{array}{c}\text { SALIDA } \\
(\mathbf{m})\end{array}$ & $\begin{array}{c}\mathbf{\Delta} \mathbf{H} \\
\mathbf{( m )}\end{array}$ \\
\hline 1 & 4,40 & 4,60 & 4,40 & 0,20 \\
\hline 2 & 14,50 & 6,60 & 6,40 & 0,20 \\
\hline 3 & 26,30 & 8,20 & 8,00 & 0,20 \\
\hline 4 & 36,70 & 8,70 & 8,40 & 0,30 \\
\hline 5 & 45,50 & 9,00 & 8,60 & 0,40 \\
\hline 7 & 54,60 & 6,00 & 5,40 & 0,60 \\
\hline 8 & 67,50 & 7,40 & 6,60 & 0,80 \\
\hline
\end{tabular}


Tabla 5. Pérdidas de carga en tubo de venturi de 1

\begin{tabular}{|c|c|c|c|c|c|}
\hline \multirow[b]{2}{*}{ No } & \multicolumn{2}{|c|}{ PRESIÓN } & \multirow{2}{*}{$\begin{array}{c}\text { CAUDAL } \\
\text { BOMBA } \\
(1 / \mathrm{min})\end{array}$} & \multirow{2}{*}{$\begin{array}{l}\Delta H \\
(\mathbf{m})\end{array}$} & \multirow{2}{*}{$\begin{array}{c}\text { CAUDAL } \\
\text { SUCCIÓN } \\
(\mathrm{l} / \mathrm{hr})\end{array}$} \\
\hline & $\begin{array}{c}\text { ENTRADA } \\
(\mathrm{m})\end{array}$ & $\begin{array}{c}\text { SALIDA } \\
(\mathrm{m})\end{array}$ & & & \\
\hline 1 & 10 & 3,0 & 41,4 & 7,00 & 10,65 \\
\hline 2 & 11 & 2,8 & 39,9 & 8,20 & 29,27 \\
\hline 3 & 12 & 2,6 & 38,2 & 9,40 & 51,67 \\
\hline 4 & 14 & 2,2 & 34,8 & 11,80 & 101,41 \\
\hline 5 & 16 & 1,8 & 31,4 & 14,20 & 136,71 \\
\hline 6 & 18 & 1,4 & 28,2 & 16,60 & 130,91 \\
\hline 7 & 20 & 0,8 & 24,9 & 19,20 & 131,71 \\
\hline 8 & 22 & 0,6 & 22,0 & 21,40 & 130,51 \\
\hline 9 & 28 & 0,0 & 14,6 & 28,00 & 129,34 \\
\hline
\end{tabular}

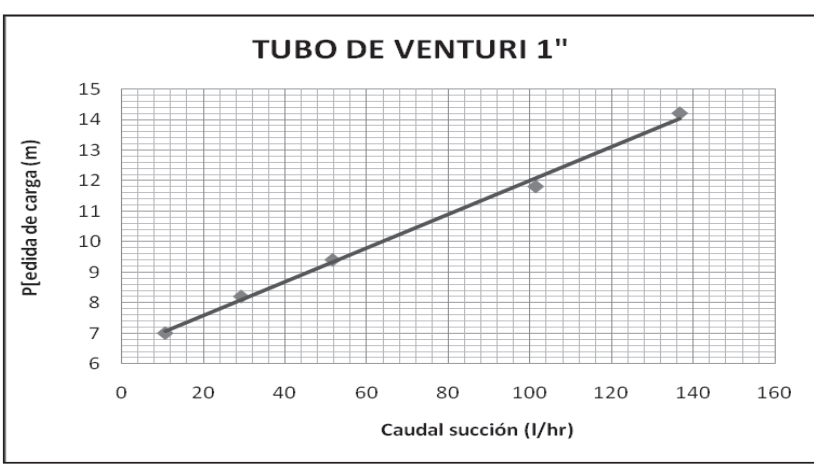

Figura 6. Curva de pérdida de carga vs caudal de succión. Tubo de venturi 1".

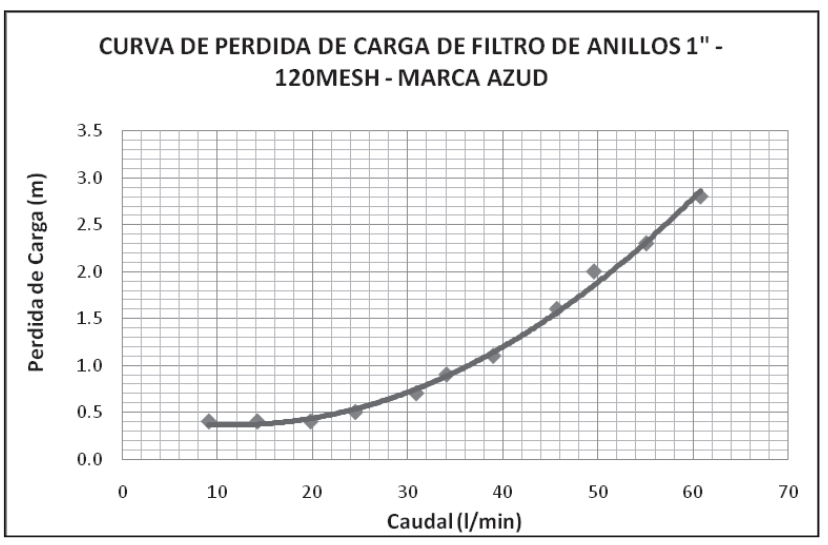

Figura 7. Curva de pérdida de carga en filtro de anillos 1 " de 120mesh.

En la Fig. 6 se muestra la curva de variación de pérdidas de carga para diferentes caudales de succión del tubo de venturi de 1". La curva generada por el venturi tiene una tendencia lineal con valor de $\mathrm{R}^{2}=0,9962$, regida por la ecuación: $\Delta \mathrm{H}=0,0553 . \mathrm{Q}+6,4716$.

\section{Filtro de anillos de 1"}

Se evaluó la pérdida de carga entre la entrada y la salida del filtro de anillos de 1" de 120mesh variando los caudales desde los 9,10 1/min hasta los 60,80 1/min, obteniendo diferentes valores de pérdida de carga.
La Fig. 7 muestra las curvas de variación de pérdidas de carga versus el caudal en el filtro de anillos de 1". Las curva generada por el filtro tiene una tendencia polinomial de segundo grado con valor de $\mathrm{R}^{2}=0,9953$, regida por la ecuación: $\Delta \mathrm{H}=0,001 . \mathrm{Q}^{2}-0,241 \mathrm{Q}+0,5054$.

\section{Línea de evaluación $V$}

Se evaluaron las pérdidas de carga por singularidad en los siguientes accesorios de PVC: codo de $3 / 4 "$ " $90^{\circ}$, codo de $3 / 4$ " $x 45^{\circ}$, tee de $3 / 4$ " y válvula esférica de $3 / 4$ ". Se evaluó también las pérdidas de carga en redes en serie y en paralelo en las que interactúan varios accesorios a la vez en cada tramo (ver Fig. 8). Los resultados obtenidos se presentan en la Tabla 6 .

\section{Red de tuberías en serie}

A los datos de la Tabla 7, se sumaron los valores de pérdida de carga de las singularidades que comprenden el tramo. El valor total de la pérdida de carga obtenida de la suma de cada singularidad, con el valor de pérdida de carga obtenida directamente de los puntos de prueba de presión, observando diferencias por errores de lectura, y la precisión de los manómetros utilizados.

\section{Red de tuberías en paralelo}

El caudal de entrada en la línea de conducción $\mathrm{V}\left(\mathrm{Q}_{\mathrm{T}}\right)$, ver Fig. 1, se dividió en dos caudales diferentes entre los tramos $\mathrm{AB}_{1}$ (con un caudal de $\mathrm{Q}_{\mathrm{AB} 1}$ ) y $\mathrm{AB}_{2}$ (con un caudal de $\mathrm{Q}_{\mathrm{AB} 2}$ ); en donde $\mathrm{Q}_{\mathrm{T}}=\mathrm{Q}_{\mathrm{AB} 1}+\mathrm{Q}_{\mathrm{AB} 2}$. Las pérdidas de carga en ambos tramos son iguales. El tramo $\mathrm{AB}_{1}$, conformado por dos codos de $3 / 4 "$ x $90^{\circ}$ y una válvula esférica de $3 / 4$ ", fue afectado por las pérdidas de carga que generaron estos tres elementos, además de la pérdida de carga generada también en las tee de $3 / 4$ " que divide y junta los caudales al inicio y al final de la red, respectivamente. El tramo $\mathrm{AB}_{2}$, conformado por dos codos de $3 / 4$ " x $90^{\circ} \mathrm{y}$ un niple PVC de $3 / 4$ " x 1,20m fue afectado por las pérdidas de carga que generaron estos tres elementos, además de la pérdida de carga generada también en las tee de $3 / 4$ " que dividen y juntan los caudales al inicio y al final de la red, respectivamente. El tramo $\mathrm{AB}_{1}$ siempre generará mayor pérdida de carga que el tramo $\mathrm{AB}_{2}$ cuando el caudal que circule por ellos sean iguales, en este caso, 
Tabla 6. Pérdidas de carga en singularidades

\begin{tabular}{|c|c|c|c|c|c|c|c|c|c|c|}
\hline \multirow[b]{2}{*}{ No } & \multicolumn{2}{|c|}{ CAUDAL } & \multicolumn{8}{|c|}{ AH (PÉRDIDA DE CARGA) } \\
\hline & $(1 / \min )$ & $\begin{array}{c}\text { VELOCIDAD } \\
(\mathrm{m} / \mathrm{s})\end{array}$ & $\begin{array}{l}\mathrm{AB} \\
(\mathrm{m})\end{array}$ & $\begin{array}{c}\text { Codo } \\
1 \\
\text { (m) }\end{array}$ & $\begin{array}{c}\text { Codo } \\
2 \\
(\mathrm{~m})\end{array}$ & $\begin{array}{l}\text { DF } \\
(\mathrm{m})\end{array}$ & $\begin{array}{c}\text { Válvula } \\
\text { (m) }\end{array}$ & $\begin{array}{c}\text { Codo3 } \\
\text { (m) }\end{array}$ & $\begin{array}{l}\text { JK } \\
(\mathbf{m})\end{array}$ & $\begin{array}{l}\text { BK } \\
\text { (m) }\end{array}$ \\
\hline 1 & 24,60 & 1,00 & 0,00 & 0,10 & 0,10 & 0,20 & 0,00 & 0,00 & 0,10 & 0,60 \\
\hline 2 & 28,70 & 1,16 & 0,10 & 0,20 & 0,20 & 0,30 & 0,00 & 0,00 & 0,20 & 0,80 \\
\hline 3 & 31,20 & 1,26 & 0,20 & 0,30 & 0,30 & 0,30 & 0,00 & 0,00 & 0,20 & 1,00 \\
\hline 5 & 4820 & 1,95 & 0,40 & 0,70 & 0,70 & 0,90 & 0,30 & 0,10 & 1,00 & 4,30 \\
\hline 6 & 51,40 & 2,08 & 0,60 & 0,80 & 0,80 & 1,10 & 0,30 & 0,10 & 1,10 & 5,00 \\
\hline
\end{tabular}

Tabla 7. Pérdida de carga en tuberías y accesorios en serie

\begin{tabular}{|c|c|c|c|c|c|c|c|c|}
\hline \multirow[b]{2}{*}{ No } & \multirow[b]{2}{*}{$\begin{array}{c}\text { CAUDAL } \\
\text { (1/min) }\end{array}$} & \multicolumn{5}{|c|}{$\begin{array}{c}\text { AH (PÉRDIDA DE CARGA EN } \\
\text { METROS) }\end{array}$} & \multirow[b]{2}{*}{$\begin{array}{c}\text { Suma total } \\
\qquad \mathbf{H} \\
\text { (m) }\end{array}$} & \multirow{2}{*}{$\begin{array}{c}\text { Lectura } \\
\text { Directa } \\
\Delta \mathbf{H} \\
\text { (m) }\end{array}$} \\
\hline & & $\begin{array}{c}03 \\
\text { codos } \\
3 / 4 ” \\
90^{\circ}\end{array}$ & $\begin{array}{c}01 \text { Tee } \\
\text { 3/4" } \\
\text { DF }\end{array}$ & $\begin{array}{c}\text { 01 } \\
\text { Válvula } \\
\text { 3/4” } \\
\text { GI }\end{array}$ & $\begin{array}{c}01 \\
\text { codo } \\
3 / 4^{\prime} \\
45^{\circ}\end{array}$ & JK & & \\
\hline 1 & 24,60 & 0,30 & 0,20 & 0,00 & 000 & 0,10 & 0,60 & 0,60 \\
\hline 2 & 28,70 & 0,60 & 0,30 & 0,00 & 0,00 & 0,20 & 1,10 & 0,80 \\
\hline 3 & 31,20 & 0,90 & 0,30 & 0,00 & 000 & 0,20 & 1,40 & 1,00 \\
\hline 5 & 48,20 & 2,10 & 0,90 & 0,30 & 0,10 & 1,00 & 4,40 & 4,30 \\
\hline 6 & 51,40 & 2,40 & 1,10 & 0,30 & 0,10 & 1,10 & 5,00 & 5,00 \\
\hline
\end{tabular}

dada la configuración entre ambos tramos, los caudales que circulan independientemente a través de cada uno de ellos son diferentes en donde $\mathrm{Q}_{\mathrm{AB} 1}<\mathrm{Q}_{\mathrm{AB} 2}$ dando como resultado pérdidas de carga iguales.

\section{Conclusiones}

Comparando las lecturas de pérdidas de carga por fricción obtenidas experimentalmente, se observa que son similares a las pérdidas de carga teóricas obtenidas del cálculo de fórmulas empíricas: Darcy-Weisbach, Hazen-Williams y Blasius; sin embargo, para determinar cuál de las formulas se acerca más a las pérdidas de carga obtenidas experimentalmente, usamos la fórmula de equilibrio de NASH, obteniendo que la fórmula de Darcy-Weisbach presenta una mejor aproximación para las tuberías de $3 / 4$ “ de PVC.

La curva generada por la válvula completamente abierta obedece a la ecuación: $\Delta \mathrm{H}=0,0226$.Q-0,2816 con $\mathrm{R}^{2}=0,9869$, la válvula semiabierta presenta una tendencia polinomial de segundo grado $\Delta \mathrm{H}=0,0008$. $\mathrm{Q}^{2}-0,0064 . \mathrm{Q}+0.1092$ con $\mathrm{R}^{2}=0,9936$, siendo mejor presentar funciones de pérdida de carga y caudal de las singularidades (filtros, válvulas, codos), que presentar coeficientes por singularidad.

El movimiento de agua se realiza de un punto de mayor energía a menor energía, y no de un punto de mayor valor piezométrico a menor valor piezométrico, tal como lo muestra la práctica de las tuberías que presentan expansiones y contracciones. Las válvulas hidráulicas reductoras y sostenedoras de presión con piloto, previamente calibradas, nos permiten reducir o sostener la presión automáticamente en una red de riego. Las pérdidas de carga del venturi son altas, siendo necesario, para su uso, la calibración con respecto al caudal de la bomba, las pérdidas de carga y el caudal de succión del venturi.

En la red en serie se comprueba que la suma de las pérdidas de las singularidades son acumulativas para un caudal determinado. En la red en paralelo se comprueba que el $\mathrm{Q}_{\mathrm{T}}=\mathrm{Q}_{\mathrm{AB} 1}+\mathrm{Q}_{\mathrm{AB} 2}$, $\mathrm{y}$ que las pérdidas de carga en ambos tramos son iguales.

El banco de prueba nos permitirá entender el comportamiento hidráulico de los elementos de una red de riego, a través del manipuleo e interacción durante las prácticas con el mismo. El costo del banco es $\mathrm{S} /$. $5.500,00$ incluida la mano de obra, con la ventaja que será un banco personalizado de acuerdo a las necesidades y cursos dictados por cada universidad. El costo de un banco de prueba en el mercado varía entre 15 mil a 20 mil nuevos soles. 


\section{Literatura citada}

Arviza Valverde, J.; Balbastre Peralta, I.; Gonzales Altozano, P. 2002. Universidad Politécnica de Valencia, España.

Biological and Irrigation Engineering Department, Utah State University. 2004. Sprinkler and trickle Irrigation Notes, fall semester. Utah, USA: Logan.

Dvir, Y. 1996. Flow Control Devices, Control Appliances books. Israel.

EPANET. 2000. Environmental Protection Agency.
USA.

Pizarro, F. 1996. Riegos localizados de alta frecuencia (goteo, micro aspersión, exudación. ( $3^{\circ}$ edición). Madrid, España: Ediciones Multiprensa.

Santos Pereira, L. 2010. El Riego y sus Tecnologías. (1 edición). España: Centro Regional de Estudios del Agua, Universidad Castilla La Mancha

Tyler, G. Hicks. 1970. Bombas su selección y aplicación. México. 